\title{
Immunoglobulin G4-Related Disease of the Orbit: Imaging Features in 27 Patients
}

\author{
C.A. Tiegs-Heiden, L.J. Eckel, C.H. Hunt, F.E. Diehn, K.M. Schwartz, D.F. Kallmes, D.R. Salomão, T.E. Witzig, and J.A. Garrity
}

\begin{abstract}
BACKGROUND AND PURPOSE: Immunoglobulin G4-related disease is a systemic fibroinflammatory process of unknown etiology, characterized by tissue infiltration by immunoglobulin G4 plasma cells. The purpose of this study was to retrospectively identify the spectrum of imaging features seen in immunoglobulin G4-related disease of the orbit.
\end{abstract}

MATERIALS AND METHODS: This study included 27 patients with biopsy-proved immunoglobulin G4-related disease of the orbit and either a CT or MR imaging of the orbits. These CT or MR imaging examinations were evaluated for the following: extraocular muscle size, extraocular muscle tendon enlargement, lacrimal gland enlargement, infiltrative process in the orbital fat (increased attenuation on CT or abnormal signal on MR imaging), infraorbital nerve enlargement, mucosal thickening in the paranasal sinuses, and extension of orbital findings intracranially.

RESULTS: Extraocular muscles were enlarged in 24 of 27 (89\%) patients, 21 (88\%) bilaterally. In 32 of 45 (71\%) affected orbits, the lateral rectus was the most enlarged muscle. In 26 (96\%) patients, the tendons of the extraocular muscles were spared. Nineteen (70\%) patients had lacrimal gland enlargement. Twelve (44\%) patients had an infiltrative process within the orbital fat. Infraorbital nerve enlargement was seen in 8 (30\%) patients. Twenty-four (89\%) patients had sinus disease. Cavernous sinus or Meckel cave extension was seen in 3 (11\%) patients.

CONCLUSIONS: In patients with extraocular muscle enlargement, particularly when the tendons are spared and the lateral rectus is the most enlarged, and even more so when other noted findings are present, immunoglobulin G4-related disease should be a leading differential consideration, even over more commonly known etiologies of extraocular muscle enlargement.

ABBREVIATIONS: IgG4 = immunoglobulin G4

l

mmunoglobulin G4 (IgG4)-related disease is a systemic inflammatory process of unknown etiology, characterized by tissue infiltration by IgG4 plasma cells and sclerosing inflammation. ${ }^{1-5}$ Although initially described in association with autoimmune pancreatitis, manifestations of IgG4-related disease are now reported in nearly every organ system. ${ }^{1-3,5-10}$

Multiple case reports and small case series of orbital manifestations of IgG4-related disease have noted involvement of the extraocular muscles, lacrimal glands, and infraorbital nerve, ${ }^{1,2,4,7-19}$

Received September 9, 2013; accepted after revision November 22.

From the Departments of Radiology (C.A.T.-H., L.J.E., C.H.H., F.E.D., K.M.S., D.F.K.), Pathology (D.R.S.), Hematology (T.E.W.), and Ophthalmology (D.R.S., J.A.G.), Mayo Clinic, Rochester, Minnesota.

Paper previously presented at: Annual Meeting of the American Society of Head and Neck Radiology, October 3-7, 2012; Miami Beach, Florida.

Please address correspondence to Laurence J. Eckel, MD, Department of Radiology, Mayo Clinic, 200 1st St SW, Rochester, Minnesota, 55905; e-mail: eckel.

laurence@mayo.edu.

http://dx.doi.org/10.3174/ajnr.A3865 but these small series do not allow evaluation of the typical patterns of imaging findings in IgG4-related disease. The purpose of this study was to retrospectively identify the spectrum of imaging features seen in IgG4-related disease of the orbit. Ideally, these characteristics will help clinicians and radiologists recognize IgG4-related orbital disease among a broad differential of orbital pathologies.

\section{MATERIALS AND METHODS}

Institutional review board approval was obtained for this Health Insurance Portability and Accountability Act-compliant retrospective research protocol, and the requirement for informed patient consent was waived. A pre-existing ophthalmology data base of all patients seen at our institution with biopsy-proved IgG4related disease of the orbit was reviewed. Patients were seen in our Ophthalmology Department between January 1998 and April 2012. All patients in this data base who had undergone CT and/or MR imaging that included the orbits were included in this study. There were no exclusion criteria aside from lack of CT and/or MR 


\begin{tabular}{|c|c|c|c|c|c|c|}
\hline & $\begin{array}{l}\text { Lateral } \\
\text { Rectus }\end{array}$ & $\begin{array}{l}\text { Inferior } \\
\text { Rectus }\end{array}$ & $\begin{array}{l}\text { Medial } \\
\text { Rectus }\end{array}$ & $\begin{array}{c}\text { Superior } \\
\text { Rectus }\end{array}$ & $\begin{array}{l}\text { Inferior } \\
\text { Oblique }\end{array}$ & $\begin{array}{l}\text { Superior } \\
\text { Oblique }\end{array}$ \\
\hline Average maximum diameter $(\mathrm{mm})^{\mathrm{a}}$ & 11.7 & 11.7 & 10.5 & 10.0 & 10.6 & 6.4 \\
\hline Average short-axis diameter $(\mathrm{mm})^{\mathrm{a}}$ & 6.4 & 6.4 & 5.2 & 5.5 & 3.0 & 3.3 \\
\hline Frequency of enlargement $\mathrm{t}^{\mathrm{b}}$ & $41(76 \%)$ & $19(35 \%)$ & $16(30 \%)$ & $11(20 \%)$ & N/A & $6(11 \%)$ \\
\hline Frequency of most enlarged ${ }^{c}$ & $32(71 \%)$ & $9(20 \%)$ & $3(7 \%)$ & $1(2 \%)$ & N/A & $0(0 \%)$ \\
\hline
\end{tabular}

${ }^{a}$ Average diameters are based on measurements from all orbits $(n=54)$.

${ }^{b}$ Frequency of enlargement was determined by the number of times a muscle was enlarged of a possible 54 orbits.

c Frequency of most enlarged muscle was determined by the number of times a muscle was the most enlarged muscle in an eye of 45 affected orbits.

imaging. Twenty-seven patients were identified for inclusion in this study. There were 17 (63\%) female patients. Mean age at the time of the initial imaging examination was 51 years (range, $30-71$ years).

Orbital biopsies were performed at our or, less commonly, an outside institution between December 1997 and April 2012. All pathology was reviewed at our institution. Biopsies were performed of the lacrimal gland, extraocular muscle, orbital softtissue infiltrate, and/or infraorbital nerve. Similar to the report of Plaza et al, ${ }^{7}$ diagnosis of IgG4 disease was made on the basis of the number of immunohistochemically identified IgG4-positive plasma cells per high-power field. For each specimen, 3 highpower fields with the highest attenuation of IgG4-positive plasma cells were selected, and an average number of IgG4-positive plasma cells per high-power field were calculated. Eleven or more IgG4 cells per high-power field were necessary to make the diagnosis of IgG4-related disease.

CT or MR imaging scans were obtained from January 1998 to December 2011. Twenty-two (81\%) patients had CT as their initial imaging examination; 5 (19\%) patients had MR imaging. CT scans were either contrast-enhanced $(12,55 \%)$ or unenhanced (10, 45\%). Section thickness of the CT scans ranged from 1.25 to $5 \mathrm{~mm}$, with most CT scans having a section thickness of $2-3 \mathrm{~mm}$ (17, 77\%). All 5 of the MR imaging examinations were performed with and without IV gadolinium. Section thickness of the MR imaging scans ranged from 3 to $5 \mathrm{~mm}$. Twenty-two (81\%) patients had their first CT or MR imaging before their biopsy. The time between the first CT or MR imaging and biopsy was $<1$ month in 16 patients (59\%), 1-2 months in 4 (15\%), 2 months to 1 year in $2(7 \%), 1-2$ years in 3 patients $(11 \%)$, and $>2$ years apart in 2 patients $(7 \%)$.

For each patient, if more than 1 study was available, the earliest CT or MR imaging was evaluated by 2 neuroradiologists ( 12 and 8 years of imaging experience) and 1 Postgraduate Year 3 radiology resident in consensus. From the coronal imaging plane, each extraocular muscle was measured in 2 dimensions (millimeters): maximum diameter and maximum short axis. Short-axis extraocular muscle measurements were compared with normal values as described by Ozgen and Ariyurek ${ }^{20}$ and Ozgen and Aydingöz. ${ }^{21}$ Upper limits of normal used for each muscle were as follows: medial rectus, $5.0 \mathrm{~mm}$; lateral rectus, $4.8 \mathrm{~mm}$; inferior rectus, 6.5 $\mathrm{mm}$; superior rectus, $6.1 \mathrm{~mm}$; and superior oblique, $4.1 \mathrm{~mm} .^{20,21}$ This reference does not provide a normal size for the inferior oblique muscle; however, this muscle was measured in a similar fashion. In addition, the examinations were subjectively evaluated for the presence or absence of extraocular muscle tendon involvement and lacrimal gland enlargement (including whether these distorted the globe or caused bony changes), the presence of an infiltrative process in the orbital fat (defined as increased attenuation on CT or abnormal signal on MR imaging in the intraconal or extraconal fat), the presence of infraorbital nerve enlargement (defined as enlargement of the infraorbital canal by CT and enlargement of the nerve by MR imaging), intracranial involvement (defined as a soft-tissue mass extending intracranially from the orbit), and incidental sinus disease (defined as fluid and/or mucosal thickening in any of the paranasal sinuses). Clinical and laboratory data, including age, sex, steroid use, serum IgG4 level (at our institution a normal IgG4 level is $2.4-121.0 \mathrm{mg} / \mathrm{dL}$ ), clinical symptoms, duration of symptoms until the first imaging study, and any other areas of known disease involvement (both within and outside the head and neck), were identified by review of the electronic medical record.

\section{RESULTS}

\section{Clinical Data}

The most frequent presenting symptom was proptosis and/or periorbital swelling $(26,96 \%)$. Pain or discomfort was reported in $2(7 \%)$ patients. Information regarding the duration of orbital symptoms was available by chart review in $24(89 \%)$ patients. The duration of symptoms ranged from 0.5 to 228.0 months (average, 44.9 months). Four (15\%) patients were taking oral steroids at the time of their initial imaging examination.

Seventeen $(63 \%)$ patients had serum IgG4 levels measured during the course of their care, some on multiple occasions. Eight of $17(47 \%)$ patients had an elevated serum IgG4 level recorded during this time. When we used only the earliest IgG4 level recorded, the average IgG4 level was $363 \mathrm{mg} / \mathrm{dL}$, with a range from 13.3 to $3150 \mathrm{mg} / \mathrm{dL}$. On the basis of this first available serum IgG4 level, $6(35 \%)$ patients had elevated values and 11 (65\%) had normal values. Serum IgG4 levels were not necessarily obtained at the time of either the initial diagnosis or initial imaging, however; in many cases, the first serum IgG4 level was not obtained until months or years after the diagnosis had been made, when the patient was already being treated.

\section{Extraocular Muscle Involvement}

The average maximal and the average short-axis diameters of each extraocular muscle (considering all 54 orbits) are shown in the Table. On the basis of an individual comparison of short-axis measurements with the aforementioned normal measurements, 24 of $27(89 \%)$ patients and 45 of 54 (83\%) orbits displayed extraocular muscle enlargement. Twenty-one of 24 (88\%) patients with extraocular muscle involvement demonstrated bilateral enlargement. Examples of unilateral and bilateral extraocular muscle enlargement are shown in Fig 1. The tendons of the extraocular muscles were spared in $26(96 \%)$ patients. 


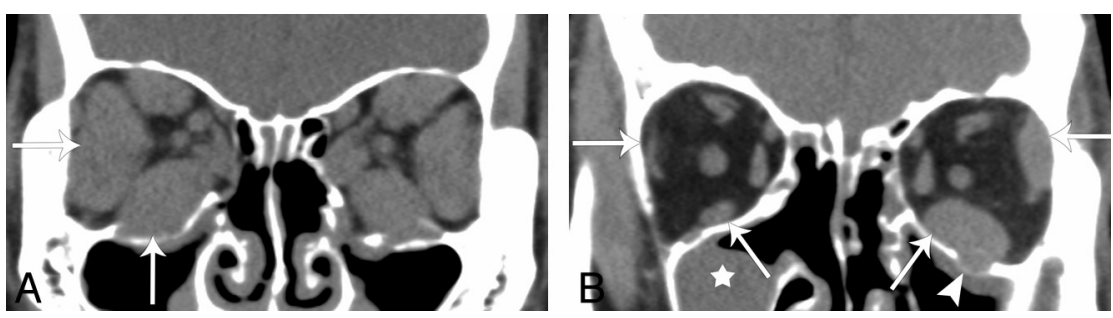

FIG 1. Noncontrast CT findings of IgG4-related ophthalmic disease. A, Bilaterally symmetric extraocular muscle enlargement in a 39-year-old man (arrows). Note that the lateral rectus is the most enlarged extraocular muscle bilaterally. B. Asymmetric left-more-than-right extraocular muscle enlargement in a 67-year-old man (arrows). Sinus disease (star) and infraorbital nerve enlargement (arrowhead) are also evident in this patient.
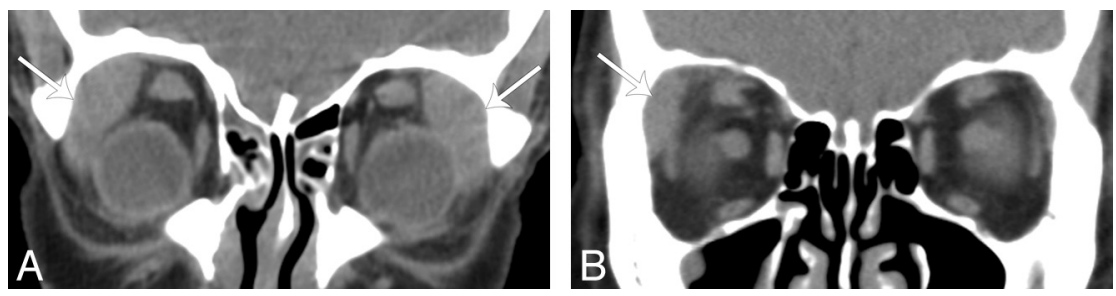

FIG 2. Lacrimal gland involvement in IgG4-related ophthalmic disease. A, Bilateral lacrimal gland enlargement in a 63-year-old woman (arrows). B, Unilateral lacrimal gland enlargement in a 59year-old woman (arrow). This patient did not have extraocular muscle enlargement.
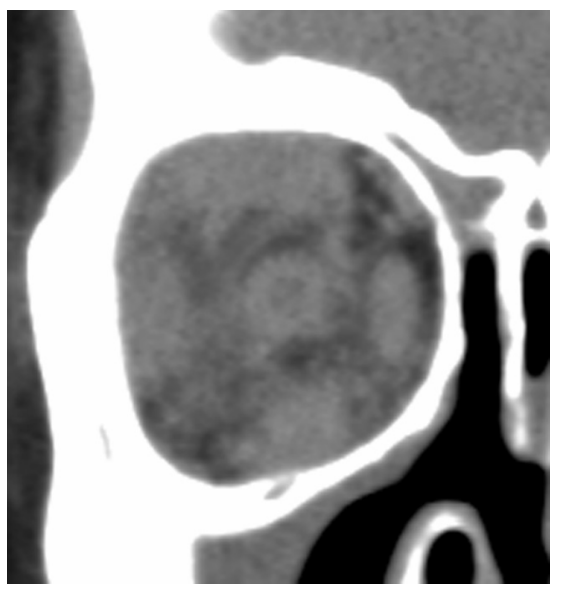

FIG 3. Infiltrative orbital process in a 42-year-old woman with IgG4related ophthalmic disease.

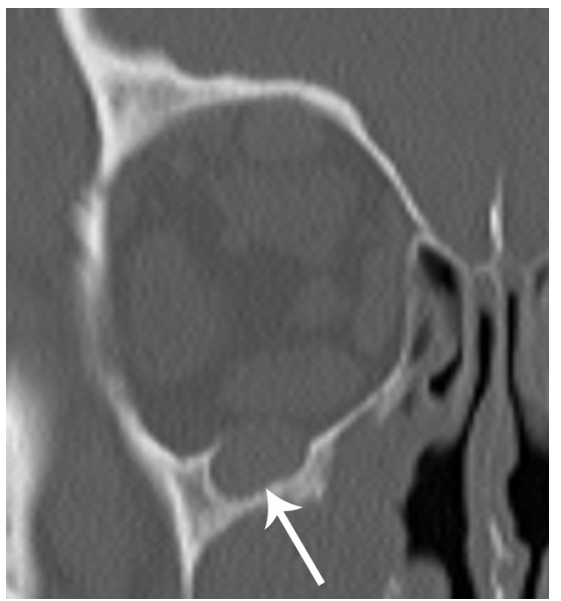

FIG 4. Infraorbital nerve enlargement in a 54-year-old man with IgG4-related ophthalmic disease (arrow). strated in Fig 1.

\section{DISCUSSION}

The Table also lists how often each extraocular muscle was enlarged compared with normal values, as well as the frequency of each extraocular muscle being the most enlarged muscle on a per-orbit basis. The lateral rectus was enlarged most commonly, in 41 of 54 (76\%) orbits. The lateral rectus was also most frequently the largest muscle in 32 of $45(71 \%)$ orbits that had extraocular muscle enlargement.

\section{Additional Imaging Features}

Nineteen (70\%) patients had lacrimal gland enlargement, 11 (58\%) of whom had bilateral involvement. Examples of unilateral and bilateral lacrimal gland enlargement are shown in Fig 2. In all cases, lacrimal gland enlargement did not distort the globe, and there were no adjacent bony changes. Sixteen (60\%) patients had both extraocular muscle enlargement and lacrimal gland enlargement. All 3 patients who did not have extraocular muscle enlargement demonstrated lacrimal gland involvement, 1 bilaterally and 2 with unilateral involvement.

Twelve (44\%) patients were characterized as having an infiltrative process within the orbital fat (Fig 3). Eight (30\%) patients had infraorbital nerve enlargement (Figs $1 B$ and 4), 5 (63\%) of which were bilateral. Three (11\%) patients had intracranial IgG4 involvement, 1 bilateral and 2 unilateral (Fig 5). Both patients with unilateral involvement had unilateral soft-tissue masses within the cavernous sinus. The patient with bilateral involvement had soft-tissue masses within the cavernous sinuses and anterior Meckel cave. Fifteen (63\%) patients had documented IgG4-related lesions outside the orbit, 11 (73\%) of which were outside the head and neck. Other areas of involvement documented in the electronic medical record included lymph nodes (cervical, thoracic, and abdominal), parotid glands, autoimmune pancreatitis, and hepatic pseudotumor. Paranasal sinus mucosal thickening was seen in 24 (89\%) patients, as demon-

On the basis of this study of the imaging of 27 patients, typical imaging findings of IgG4-related disease of the orbit are evident. The most common orbital imaging finding in our patient population was extraocular muscle enlargement. The lateral rectus was the most commonly enlarged muscle and was typically enlarged to the greatest degree. Lacrimal gland enlargement was also a very common finding. Intraorbital inflammatory change, infraorbital nerve enlargement, and sinus disease are supportive findings in IgG4-related disease. While uncommon, a soft-tissue mass extending from the posterior orbit into the cavernous sinus and/or Meckel cave may also be noted. The combination of any primary (extraocular muscle enlargement or lacrimal gland enlargement) 
and any of these other supportive findings is very suggestive of IgG4 disease (Fig 6).

The inflammatory mucosal thickening noted in the paranasal sinuses is an unknown association with IgG4-related disease of the orbit. Given that it was noted in $89 \%$ of our patients versus a prevalence of approximately $40 \%$ in the general population, ${ }^{22}$ perhaps it reflects another manifestation of altered immune modulation in those patients with IgG4-related disease. As such, its presence may offer an additional clue when considering IgG4 of the orbit versus other etiologies.

The extraocular muscle enlargement seen in many patients in this series could be misinterpreted as being secondary to Graves ophthalmopathy. A useful distinguishing feature between these 2 entities is the pattern of extraocular muscle involvement. Graves ophthalmopathy tends to spare the lateral rectus until late in the disease course. ${ }^{23,24}$ By contrast, in our study, the lateral rectus is the most commonly and most dramatically involved in IgG4related disease. Additionally, findings of sinus disease and infraor-
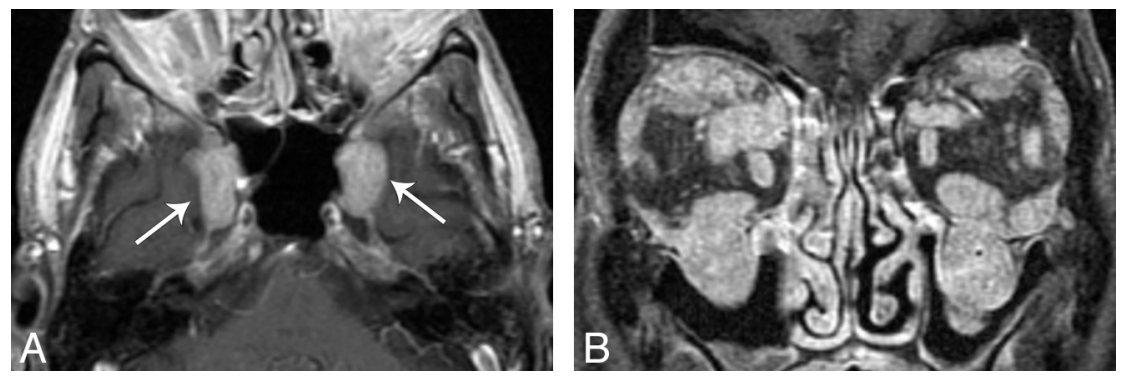

FIG 5. Intracranial disease in IgG4-related ophthalmic disease. A, Axial Tl postcontrast image in a 70-year-old man demonstrates enhancing soft-tissue masses extending bilaterally through the cavernous sinus to the anterior Meckel caves (arrows). B, Coronal Tl postcontrast image in the same patient demonstrates extraocular muscle enlargement, infraorbital nerve enlargement, and sinus disease. bital nerve enlargement are also not usual features of Graves ophthalmopathy. ${ }^{23}$

Idiopathic inflammatory orbital pseudotumor could also be included in the differential diagnosis of IgG4-related disease of the orbit. Orbital pseudotumor can present with dacryoadenitis, myositis, apical, anterior, or diffuse disease. ${ }^{25}$ Whereas IgG4 typically spares the tendinous insertions of the extraocular muscles, orbital myositis characteristically causes enlargement of the entire muscle, including the tendon. ${ }^{25}$ Again, the pattern of extraocular muscle enlargement is an important differentiating feature. Orbital myositis most frequently affects the medial rectus, followed by the superior rectus and lateral rectus, ${ }^{25}$ and is most often unilateral. ${ }^{25}$ In contrast, IgG4 favors the lateral rectus and is most commonly bilateral. Unlike IgG4-related disease of the orbit, patients with orbital pseudotumor classically present with orbital pain. $^{25}$

This case series is, to our knowledge, the largest to describe the pattern of imaging findings seen in IgG4-related disease of the orbit. A recent series of 9 cases also identified lacrimal gland enlargement, extraocular muscle thickening, orbital fat involvement, and perineural involvement as imaging features of IgG4-related disease. ${ }^{19}$ While this series found all cases of extraocular muscle thickening to be unilateral, cases in our series were more often bilateral. ${ }^{19}$ Several previous publications regarding IgG4-related disease of the head and neck have focused their attention within the orbit on lacrimal gland enlargement. ${ }^{4,7,17,18}$ Although lacrimal gland enlargement was com-

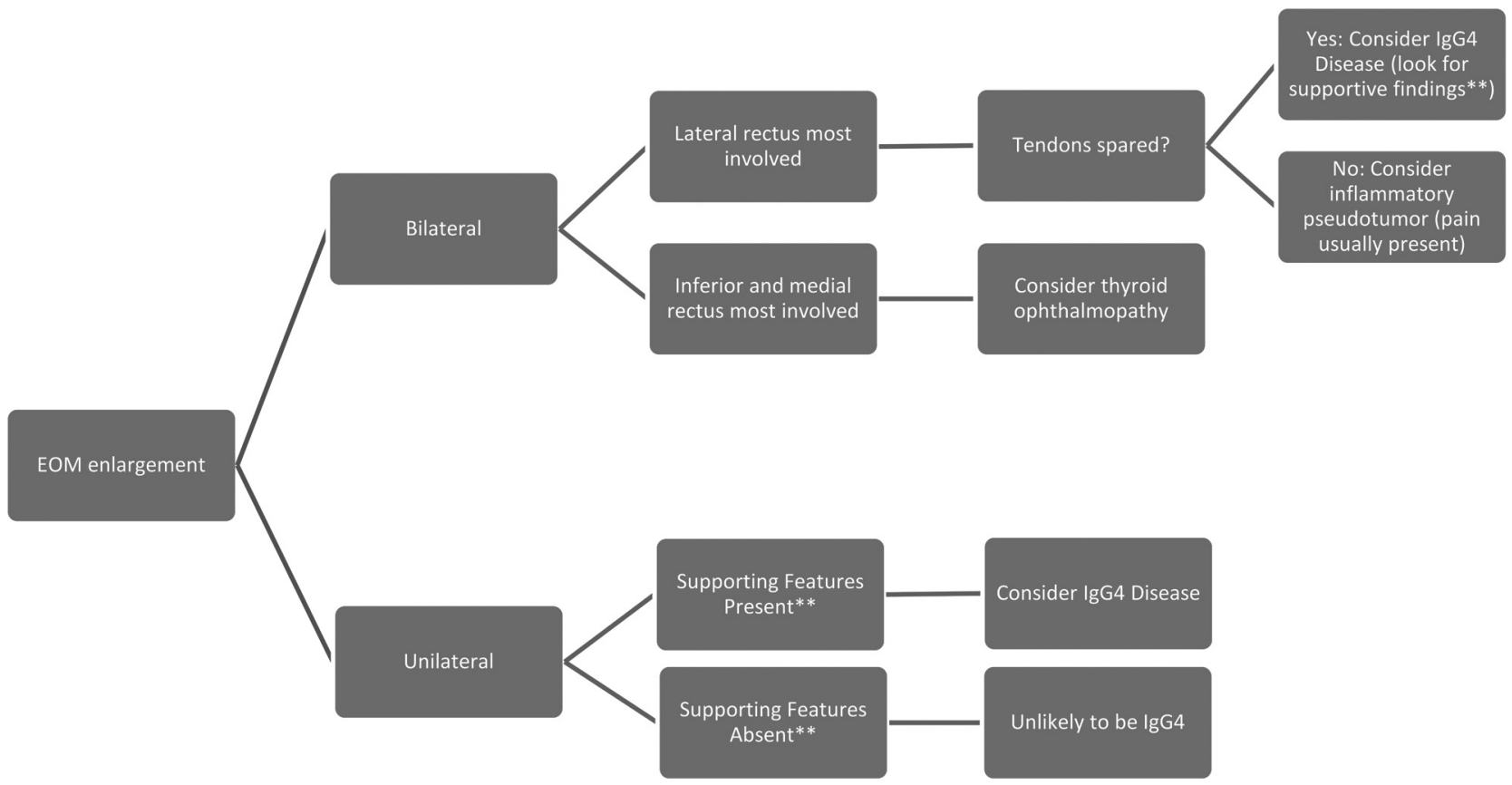

** Supporting features include: lacrimal gland involvement, sinus disease, infraorbital nerve enlargement, and/or an infiltrative process in the orbital fat.

FIG 6. Decision tree for the differential diagnosis of extraocular muscle enlargement. 
mon in this case series, extraocular muscle enlargement was the most common finding. Several authors have also reported the presence of perineural spread of IgG4-related disease, with expansion of the associated neural foramina. ${ }^{4,8,9,17,18}$

Limitations of our study include its retrospective nature. Additionally, the imaging-acquisition parameters were not consistent among all patients. The study used 2 different modalities, CT and MR imaging, because the initial examination technique differed among patients. Some scans were not dedicated orbital studies but were performed to assess the sinuses or entire head. This difference could potentially decrease the sensitivity of detection for some of the findings that were assessed.

Future research could include evaluation of imaging findings more closely coordinated to the date of systemic IgG4-level acquisition. We did not identify any published literature regarding serum IgG4 in relation to orbital manifestations. Additionally, further research to investigate changes in orbital imaging features with time would be helpful to document the suspected duration, natural course of presentation and suspected progression, and response to treatment.

\section{CONCLUSIONS}

In patients with proptosis or periorbital swelling who undergo CT or MR imaging, IgG4-related orbital disease can be strongly suspected when certain imaging features are also present. When extraocular muscle or lacrimal gland enlargement is noted, particularly when the lateral rectus is the most involved muscle, IgG4 should be a differential consideration. In these cases, if orbital inflammatory change, infraorbital nerve enlargement, or incidental sinus mucosal thickening, or all of these are also noted, IgG4related orbital disease should be a leading consideration over other more commonly known etiologies of extraocular muscle and lacrimal gland enlargement.

Disclosures: David F. Kallmes—UNRELATED: Consultancy: ev3,* Codman,* Medtronic, ${ }^{*}$ Comments: planning and implementing clinical trials, Grants/Grants Pending: MicroVention, ${ }^{\star}$ Codman, ${ }^{\star}$ ev3,, NeuroSigma, ${ }^{\star}$ Sequent Medical, ${ }^{\star}$ Benvenue Medical, ${ }^{*}$ Comments: preclinical and clinical research, Royalties: University of Virginia Patent Foundation, Comments: Spine Fusion. *Money paid to the institution.

\section{REFERENCES}

1. Cheuk W, Chan JK. IgG4-related sclerosing disease: a critical appraisal of an evolving clinicopathologic entity. Adv Anat Pathol 2010;17:303-32

2. Wallace ZS, Khosroshahi A, Jakobiec FA, et al. IgG4-related systemic disease as a cause of "idiopathic" orbital inflammation, including orbital myositis, and trigeminal nerve involvement. Surv Ophthalmol 2012;57:26-33

3. Umehara H, Okazaki K, Masaki Y, et al. Comprehensive diagnostic criteria for IgG4-related disease (IgG4-RD), 2011. Mod Rheumatol 2012;22:21-30

4. Katsura M, Mori H, Kunimatsu A, et al. Radiological features of
IgG4-related disease in the head, neck, and brain. Neuroradiology 2012;54:873-82

5. Zen Y, Nakanuma Y. IgG4-related disease: a cross-sectional study of 114 cases. Am J Surg Pathol 2010;34:1812-19

6. Fujinaga Y, Kadoya M, Hamano H, et al. Radiologic findings of IgG4-related disease. Curr Immunol Rev 2011;7:186-203

7. Plaza JA, Garrity JA, Dogan A, et al. Orbital inflammation with IgG4-positive plasma cells: manifestation of IgG4 systemic disease. Arch Ophthalmol 2011;129:421-28

8. Watanabe T, Fujinaga $\mathrm{Y}$, Kawakami S, et al. Infraorbital nerve swelling associated with autoimmune pancreatitis. Jpn J Radiol 2011; 29:194-201

9. Inoue D, Zen Y, Sato Y, et al. IgG4-related perineural disease. Int J Rheumatol 2012;2012:401890

10. Masaki Y, Dong L, Kurose N, et al. Proposal for a new clinical entity, IgG4-positive multiorgan lymphoproliferative syndrome: analysis of 64 cases of IgG4-related disorders. Ann Rheuma Dis 2009;68: $1310-15$

11. Katsura M, Morita A, Horiuchi H, et al. IgG4-related inflammatory pseudotumor of the trigeminal nerve: another component of IgG4related sclerosing disease? AJNR Am J Neuroradiol 2011;32:E150-52

12. Kubota T, Moritani S, Katayama M, et al. Ocular adnexal IgG4-related lymphoplasmacytic infiltrative disorder. Arch Ophthalmol 2010;128:577-84

13. Takahira M, Ozawa $Y$, Kawano M, et al. Clinical aspects of IgG4related .orbital inflammation in a case series of ocular adnexal lymphoproliferative disorders. Int J Rheumatol 2012;2012:635473

14. Cheuk W, Yuen HKL, Chan JKC. Chronic sclerosing dacryoadenitis: part of the spectrum of IgG4-related sclerosing disease? Am J Surg Pathol 2007;31:643-45

15. Higashiyama T, Nishida Y, Ugi S, et al. A case of extraocular muscle swelling due to IgG4-related sclerosing disease. Jpn J Ophthalmol 2011;55:315-17

16. Fonte R, Pirali B, Caramia V, et al. Graves'-like orbitopathy in a patient with chronic autoimmune pancreatitis. Thyroid 2011; 21:1389-92

17. Toyoda K, Oba H, Kutomi K, et al. MR imaging of IgG4-related disease in the head and neck and brain. AJNR Am J Neuroradiol 2012;33:2136-39

18. Fujita A, Sakai O, Chapman M, et al. IgG4-related disease of the head and neck: CT and MR imaging manifestations. Radiographics 2012;32:1945-58

19. Ginat DT, Freitag SK, Kieff D, et al. Radiographic patterns of orbital involvement in IgG4-related disease. Ophthal Plast Reconstr Surg 2013;29:261-66

20. Ozgen A, Ariyurek M. Normative measurements of orbital structures using CT. AJR Am J Roentgenol 1998;170:1093-96

21. Ozgen A, Aydingöz U. Normative measurements of orbital structures using MRI. J Comput Assist Tomogr 2000;24:493-96

22. Havas TE, Motbey JA, Gullane PJ. Prevalence of incidental abnormalities on computed tomographic scans of the paranasal sinuses. Arch Otolaryngol Head Neck Surg 1988;114:856-59

23. Kahaly GJ. Imaging in thyroid-associated orbitopathy. Eur J Endocrinol 2001;145:107-18

24. Kirsch E, Hammer B, von Arx G. Graves' orbitopathy: current imaging procedures. Swiss Med Wkly 2009;139:618-23

25. Ding ZX, Lip G, Chong V. Idiopathic orbital pseudotumour. Clin Radiol 2011;66:886-92 